\title{
A Brief Survey to Swarm Stability Problems for Swarm Systems: Part 1 Caifeng Mai ${ }^{1, a}$ and Ning Cai ${ }^{1, b^{*}}$ \\ ${ }^{1}$ College of Electrical Engineering, Northwest Minzu University, Lanzhou, China \\ a1213102363@qq.com, bcaining91@tsinghua.org.cn \\ ${ }^{*}$ The corresponding author
}

Keywords: Swarm System; Agent; Consensus; Swarm Stability; Graph

\begin{abstract}
Some analysis for the traditional isolation system has been matured and the concept of comprehensive, such as stability, balance, controllability, servo controller, etc. For the swarm systems are required to do the corresponding development. Around this issue, there are many unknown fields to need to be expanded. In essence, consensus is similar to the balance of the isolating system. It is considering the relative movement between the agents of a fundamental theoretical basis. This article will classify the important results related to this work in the literature.
\end{abstract}

\section{Introduction}

For swarm systems, consensus means that any variable in every agent asymptotically converges to a same value [1-4]. These variables depend on the states of the agents. Consensus algorithms or protocols are a kind of distributing information coordination rules, which determines how to transform information between adjacent agents, thus can make the entire swarm systems implement consensus. So far, the studies of consensus can be roughly divided into three stages, where the first two stages will be surveyed in the current paper.

\section{The First Stage (before 2004)}

Consensus issues are originated in the field of computer science. In a sense, it composed the basic theory of distributed parallel computing. Issues related to parallel computing field been consistent with the consensus's early work are summarized in [5].

It can be argued that the concept of consensus the earliest research has come into the field of dynamic systems since 1987. Reynolds created a system of the boids, and he proposed well-known rules of group system modeling for the purpose of imitating the movement of birds on the computer.

1) Repel: Due to the distance between multi-agent too close and they feel uncomfortable or dangerous to take the initiative to avoid collision with each other.

2) Speed match: Adjacent individuals will adjust the speed between each other as much as possible to maintain the same speed.

3) Attract: As long as the distance adjacent individuals are not very close, they will attract each other.

The three rules that seem simple are actually able to ensure that the speed of movement of a group of birds tends to be consistent.

Afterwards, the physicist Vicsek et al. observed the phase transition phenomenon that occurred during the movement of a particular particle system by experiment simulation. Phase transition movement of the particle group refers to the movement of the morphological changes. Vicsek has observed many different types of phase transition phenomena.

Consensus is the simplest and most fundamental phase transition, and it also is a change from disordered state to orderly state. In a particular particle system, the velocities of the random distributed particles tend to be consistent at the beginning of the time. Jadbabaie et al. tried to use mathematics to prove the phenomenon of phase transition observed by Vicsek through experiment simulation. His work may be the beginning of the consensus issues that more and more scientific and technical experts in the field of control and systems science are turning their point of view to. 
What he thought of is a system model of a discrete time swarm:

$$
\begin{aligned}
& \theta_{\mathrm{i}}(t+1)=\left\langle\theta_{i}(t)\right\rangle_{r} \\
& \left\langle\theta_{\mathrm{i}}(\mathrm{t})\right\rangle_{r}=\frac{1}{1+\left|N_{i}(t)\right|}\left(\theta_{i}(t)+\sum_{j \in N_{i}(t)} \theta_{j}(t)\right)
\end{aligned}
$$

In the above equation $\theta_{\mathrm{i}}$ represents the direction of the movement of the $\mathrm{i}$-th agent, the time-varying set $\mathrm{N}_{\mathrm{i}}(\mathrm{t})$ represents, the neighborhood of the $\mathrm{i}$-th multi-agent at that time, and $|\bullet|$ represents the cardinal of the set. The main contribution of this study is to prove that the connectivity of information channel structure is the key to achieve the consensus. The basic connectivity requires that the union of all time-varying switching graphs can form a connected graph in a sufficiently long period of time. Jadbabaie said any graph that satisfies this condition is jointly connected. Methods involved in this work are mainly algebraic and include the limit of infinite matrix product, random matrix, nonnegative matrix theory and so on. Originality of the relatively stronger work of its influence is relatively large and it main idea comes from the Markov chain problem. Since the result from Jadbabaie have been published so far, the research work on the problems of consensus of discrete time group systems in the literature has basically inherited the idea and method of article [6].

Fax and Murray studied the problem of relative motion stability of linear time-invariant homogeneous group systems. The interaction between the agents in this swarm system is achieved by a dynamic compensator. They found that the stability analysis of the relative motion of the whole swarm system is equivalent to the stability analysis of a single individual model that is modified for the Laplacian spectrum according to the graph topology. Although they did not directly discuss consensus issues, their works could serve as a basis for researching consensus. Fax and Murray first introduced the concept of the Laplacian spectrum into the work of stability analysis about swarm system. So far, most of the literature on the consensus issues of continuous-time swarm systems has inherited the basic ideas and basic approaches in [7].

\section{Second Stage (From 2004 to 2007)}

During this period, the problem of consensus has formally entered the field of automatic control and it has gradually become a hot research topic in this field. Although the results of this period are not many, most are the groundbreaking and milestone research results. The methods and ideas involved in this study always have a far-reaching impact. During this period, most of the research work is in line with the first-order swarm systems to explore the conditions for checking consensus.

Olfati-Saber and Murray first introduced the concept of consensus into the field of automatic control. They had done a lot of valuable and groundbreaking works, in which the milestone is the article [8]. The paper focuses on the problems of the average consensus of the first-order swarm systems. Olfati-Saber and Murray found that the sufficient condition for ensuring that a system achieves consensus is that the union of the switching graphs form a strongly connected graph within a sufficiently long period of time for a swarm system with time-varying and directed graph. The condition that the state of each agent exponentially converges to the average is that the graph is balanced.

Ren and Beard's research work on the problem of consensus [9] was mainly inspired by Oifati-saber and Murray's research results [8]. They discussed the problem of consensus, linear discrete-time swarm systems with switching graphs. The model is basically the same as Vicsek's: 


$$
\xi_{\mathrm{i}}(\mathrm{k}+1)=\frac{\left(\sum_{\mathrm{j}=1}^{\mathrm{N}} \alpha_{\mathrm{ij}}(\mathrm{k}) \mathrm{G}_{\mathrm{ij}}(\mathrm{k}) \xi_{\mathrm{j}}(\mathrm{k})\right)}{\sum_{\mathrm{j}=1}^{N} \alpha_{\mathrm{ij}}(\mathrm{k}) \mathrm{G}_{\mathrm{ij}}(\mathrm{k})}
$$

$\xi_{\mathrm{i}}$ is coordinate variable; $\alpha_{\mathrm{ij}}$ is the arc weights; $\mathrm{G}_{\mathrm{ij}}(\mathrm{k}) \in\{0,1\}$ is a logic quantity and indicates connection or not.

Ren and Beard also consider the problem of consistency of linear continuous time swarm systems. The model as following:

$$
\dot{\xi}_{\mathrm{i}}(\mathrm{t})=-\sum_{\mathrm{j}=1}^{\mathrm{N}} \sigma_{\mathrm{ij}}(\mathrm{t}) \mathrm{G}_{\mathrm{ij}}(\mathrm{t})\left(\xi_{\mathrm{i}}(\mathrm{t})-\xi_{\mathrm{j}}(\mathrm{t})\right)
$$

This model inherits the definition of Oifati- saber and Murray. For Ben and Beard in swarm system the most important contribution of the consensus criterion is that it relaxes the requirement of strong connectivity of the digraph in the literature [8]. They prove that the directed graph contains a spanning tree that is the necessary and sufficient conditions that can achieve consensus for the linear first order swarm system with appropriate consensus agreement. But the strong connectivity of directed graph is only a sufficient condition. The result of Ren and Beard is very important, which is recognized by everyone as the basic conclusions. Since then, in most literature of studying consistency, directed graph should include a spanning tree as premise swarm systems that have become a must following condition.

One of the most noteworthy directions in the method of all study of consistency conditions for swarm systems is Moreau whose research work is based on local convex analysis. Moreau also considers the problem of consistency swarm systems that it has general higher order nonlinear of discrete time with directed and time-varying graphs. The form of this model is more general.

$$
\left\{\begin{array}{l}
\chi_{1}(\mathrm{t}+1)=f_{1}\left(t, \chi_{1}(t), \ldots, \chi_{N}(t)\right) \\
\chi_{2}(t+1)=f_{2}\left(t, \chi_{1}(t), \ldots, \chi_{N}(t)\right) \\
\vdots \\
\chi_{N}(t+1)=f_{N}\left(t, \chi_{1}(t), \ldots, \chi_{N}(t)\right)
\end{array}\right.
$$

In order to describe the method, Moreau put forward the concept of set-valued Lyapunov Function. The value of Lyapunov function is a set, instead of the traditional non negative real numbers or vector. The conclusion of Moreau is that the system will achieve the consensus, when the discrete time of swarm system Eq.5 in accordance with the following conditions.

1) After each step of movement, the new position of the agent in the state space falls within the convex hull formed by the neighbor;

2) In a sufficiently long time, the graph formed by the union of discrete time switching graph has the sufficient connectivity.

Moreau will also study the results that are extended to continuous-time model. For continuous-time systems that are similar to Eq.5, the criterion condition for the graph should be modified that continuous time-varying directed graph formed by integral graph has sufficient connectivity in a long enough period of time. Actually, Moreau's assumptions such as 1) has the more demanding. Based on the work of Moreau, Lin and others [10] have the basis for some expansion. He relaxes the first condition, only by the agent direction of the vector field to its neighbors' convex hull.

In this period, in addition to the study on a variety of different models of the consensus conditions, there are also some other exploration work on the concept of consensus in the literature.

Olfati-saber and others notice that the linear first-order swarm systems of consensus is exponential convergence, and the convergence rate is directly related to the graph Laplacian matrix closest to zero special values [8]. They also found [11] that the convergence rate of the swarm 
systems has an information channel structure based on small world model which is very fast, and has nothing to do with the order of the graph.

Xiao and Boyd focus on how to design the structure of information channel, which makes the consensus of their swarm systems converge faster.

Hatano and Mesbahi study on the first-order swarm system of consensus based on random graph. They found that time-varying random graph can always meet the minimum connectivity conditions to achieve consensus requirements. At any time this graph whether there will be a side which is random between the two points or not. The result is worth noticing. Actually every side of the random graph, the weights in a long enough time within the expected value of integral must be greater than zero. Therefore, the integral of random graph within long time range directly can be regarded as a complete graph.

During this period, the scholars who have consistent focus on the problems of consensus and mostly are still studying on the agent model that is a first-order integrator swarm systems. The system is called the first-order swarm systems. Moreau's model is the high order and nonlinear system. But there is a default assumption in its model: After each step of movement, the new position of the agent in the state space falls within the convex hull formed by the neighbor. This assumption is not consistent with the actual situation of most of the high-order systems. Except for a few of studies [12-14] the two order model based on the specific structure, there is little work involved in higher order system models. This may have two reasons:

1) The first order integrator is very simple, and it does not involve some dynamic phenomena such as inertia, damping and shock. So, the focus of this study is to focus on the influencing of the information channel structure on the consistency of the system.

2) To a great extent, the research perspective has yet to get rid of the impact of early distributing computing background. And it is no real enter into the control and systems of science field. Coordinate variables are scalar, and application backgrounds are limited to sensor networks. The first-order integrator is only the simplest dynamical systems. Most researchers have not yet put agent of general control system for the higher-order system of consistency issues.

During this period, some scholars began to realize that the consistency may be closely related to the formation control problems, which is the theoretical basis of formation control. And they try to unify the two problems. Among them, a lot of attempts have been put into. Ren et al. have been trying to construct a theoretical framework that has unified consensus and the formation control problems [13-14]. For example, in [15], Ren et al. have defined the concept of "coordination variables". The so-called coordinated variable refers to the basic information that must be shared in order to ensure that the agent in the system collaborate to achieve the group goals. The so-called coordinated variable is not necessarily directly related to the whole state of the system. In [9], Ren et al. establish a coordination model based on consistency that coordination variables represent a kind of global information and each agent maintains a local copy of the value, the behavior of the agent to coordinate variables as reference. The copy of coordination variables on all agents should be consistent. In [15], Ren et al. tried to put the concept of consensus to different aspects of formation control. They tried to prove that some leader-follower control mode in the literature, behavior-based approach and virtual structure approach, in some sense can be regarded as a special case of formation control of basing on consistency. In addition, Ren et al. [10] earlier analysis consensus algorithm that based on the specific of two order swarm system model and expand the results of first order model.

During this period, for the problem of a first-order system of consensus, and related research the most main direct of application background is the "sensor network fusion". Many sensors are distributed in a certain space range to form a network, and together to measure some physical variable. Achieving consensus means harmonizing the different measurements values of all sensors.

\section{Conclusions}

The above is a brief introduction to the firt two stages of some of the important research work on consensus. 


\section{References}

[1] N Cai, J Cao, H Ma, C Wang, Swarm stability analysis of nonlinear dynamical multi-agent systems via relative Lyapunov function, Arab. J. Sci. Eng., 39 (2014), pp. 2427-2434.

[2] N Cai, M J Khan, Almost decouplability of any directed weighted network topology, Physica A, 436 (2015), pp. 637-645.

[3] J Xi, M He, H Liu, J Zheng, Admissible output consensualization control for singular multi-agent systems with time delays, J. Franklin Inst., 353 (2016), pp. 4074-4090.

[4] N Cai, C Diao, M J Khan, A novel clustering method based on quasi-consensus motions of dynamical multi-agent systems, Complexity, 2017, 4978613.

[5] N A Lynch: Distributed Algorithms (Morgan Kaufmann, San Mateo 1996).

[6] A Jadbabaie, J Lin, A S Morse, Coordination of groups of mobile autonomous agents using nearest neighbor rules, IEEE Trans Automal Control., 48 (2003), pp. 988-1001.

[7] J A Fax, R M Murray, Information flow and cooperative control of vehicle formations, IEEE Trans Automat Control., 49 (2004), pp. 1465-1476.

[8] R Olfati-Saber, R M Murray, Consensus problems in networks of agents with switching topology and time-delays, IEEE Trans Automat Control., 49 (2004), pp. 1520-1533.

[9] W Ren, R W Bread, Consensus seeking in multi-agent systems under dynamically changing interaction topologies, IEEE Trans Automat Control., 50 (2005), pp. 655-661.

[10] Z Lin, B Francis, M Maggiore, On the state agreement problem for multiple nonlinear dynamical systems, Proc. IFAC, (2005).

[11] R Olfati-Saber, Ultrafast consensus in small-world networks, Proc. Amer Control Conf., (USA, 2005).

[12] W Ren, Cooperative control design strategies with local interactions, Proc. IEEE Int Conf Network Sensing Control., (2006).

[13] W Ren, Consensus based formation control strategies for multi-vehicle systems, Proc. Amer Control Conf., (2006).

[14] W Ren, E M Atkins, Distributed multi-vehicle coordinated control via local information exchange, Int J Robust Nonlinear Control. 17 (2007), pp. 1002-1033.

[15] W Ren, R W Beard, T W McLain, Lecture Notes in Control and Information Science, Proc. Block Island Workshop on Cooperative Control, Springer-Verlag Series., 309, pp. 171-188. 